\title{
Insomnia and its Management- An Ayurvedic Overview
}

\section{Editorial}

Sleep is one among the best elixir and tonic of life. It is a natural rejuvenator to refresh every living being in the earth. Any alterations in the quantity, quality as well as the patterns of the sleep, contributes to various disorders of sleep. Sleep disturbances occur in many of the psychiatric illnesses and is also as a component of the diagnostic criteria for specific disorders. According to National institute of health, the prevalence of sleep disorders in America is approximately among $14.71 \%$ of the population. A higher prevalence of sleep disorders related to initiation and maintenance of sleep $(28 \%)$ was reported in an urban population from India. Among these sleep disorders, insomnia is the commonest clinical presentation which is $40 \%$ more common in women than in men. Severe insomnia is sleeping less than 3.5 hours in females and 4.5 hours in males, may lead to a reduced life span.

Insomnia is in fact, both a symptom as well as a disorder. Chronic insomnia is more prevalent than heart disease, cancer, AIDS, diabetes and several other most prevalent disorders. If left untreated, insomnia increases the risk of developing diseases like depression, diabetes, hypertension and possibly even death in older individuals as per reported studies. Therefore it is very much essential to identify and treat the condition of insomnia at the earliest.

\section{Ayurvedic approach}

In Ayurveda, Sleep or Nidra have been explained as one of the triads for sustaining life. The alteration of the same as well as etiopathogenesis along with the management is being explained in the classics in the terminology "Nidranasa." Among the three doshas controlling the human physiology, such a condition results from the aggravation of the doshas - Vatha and Pitta, and also depletion of the dosha - Kapha. Also the sleep is regulated by the performance of the dosha in relation with the functioning of manas ie. Rajas Hence it may be manifested both in physical as well as psychiatric disorders. The initiation of sleep is promoted by Vāta and the maintenance of sleep is enhanced by Pitta.

Fundamental functions of Vāta, in connection with mental business are activation (pravarttaka), controlling (niyanta) and motivation (preraka). These basic functions are impaired, while the aggravation of Vāta takes place due to specific causes in any conditions, including sleep. The clarity of the sense organs also is contributing to a normal physiologic sleep. Consequently, mano nivrtti, a relaxed state of mind is very much for a sound sleep to get ensued.

In nidranasa, the aggravation of Vāta occurs which will further vitiate the other doshas, Pitta as well as Kapha as per the etiology. Vatha and manas are very much related in their functioning. Hathayoga Pradeepika highlighted the inter-relationship of Vāta and Manas by saying that mind gets active on the light of Vatha. Vatha kopa also results in dhatukshaya which in fact may lead to depletion of the ojus and hence results in physical as well as

Volume 9 Issue 2 - 2017
Jithesh Madhavan* and Jayasree Vijayan
Department of Ayurvedic Psychiatry, VPSV Ayurevda College,
India
*Corresponding author: Jithesh Madhavan, PG Department
of Ayurvedic Psychiatry, VPSV Ayurevda College, Kottakkal,
India, Tel: 09447882885; Email: drjitheshm@gmail.com
Received: October 31, 2017 | Published: November 03,
2017

somatic ailments. Hence it is of ultimate importance to regulate Vāta, in the management of Nidranasa.

\section{Management}

Insomnia has to be approached in a very detailed manner regardless of the system of medicine, we are practicing upon. The management should be commenced with education about sleep hygiene as well as addressing the sleep problems. For the same, a detailed history of all the aspects of life style, along with sleep have to be traced out as many of the sleep disturbances are in relation with the life style of the affected.

Adequate sleep hygiene is so much essential which includes maintenance of regular hours of bed time and arousal, avoiding excessive caffeine, not ingesting heavy meals before bed time and performing adequate exercise and mental relaxations like listening to favorite music before going to bed. The day-to-day changes in behavior and changing severity of sleeplessness can obscure the factors responsible for the problem. A carefully explained program of sleep hygiene, with follow up, represents a fairly inexpensive but much effective intervention.

Pharmacotherapy includes sedative - hypnotic or melatonin receptor agonist, antidepressants, benzodiazepines, non benzodiazepine sedatives, orexin etc. Eventhough they are drugs that help to induce sleep, no one promotes the prolonged use of these medicines that may cause dependency as well as addiction in many. Adverse effects such as depression, thoughts of self injury or suicide, anxiety, aggression, restlessness, hallucinations etc are another major issue of the long term use of sedatives. So the management options in alternative medicines should be promoted in order to enhance the quality of life of the affected.

Based on the severity of the presentation, insomnias are approached to be managed at the Outpatient level in mild to moderate cases and as inpatient for the severely affected, so as to perform the sodhana ie., the purificatory therapy. The sodhana therapy starts with gritha administration, based on the selected dosha, followed by sweda and Virechana. This is followed by 
purificatory therapy of the head ie. nasya with suitable gritha or taila as per the demand from the condition. The next step is of the procedures such as śirodhāra, śirovasti, sirolepa etc. which are performed on the siras or head with suitable medicines. Utsādana ie., the gentle massage with a mixture of oil and medicated powder over the body or Samvāhanam, soft touch or gentle massage is also being done.

In the Outpatient level, several combinations are being practiced and proved as effective. The commonly used drugs are that pacifies vatha and Pitta amnd also relaxe the mind. Some of the single drugs are Tagara (Valerina Wallichi), Jatamansi (Nordostachys Jatamansi), Somalata (Sarcostemma acidum) and Aśwagandha( Withania somnifera). Talam ie the application of a selected mixture of curna (powder) and tailom (oil) on the vertex of the head is done. Siroabhyanga ie., the application of oil on head with gentle head massage is of significant use. Similar is the use of Pada abhyangam or the application of medicated oil under the feet.

The medhya rasayana groups of drugs are also of proven efficacy in the management of insomnia which includes mandookaparni (Centella asiatica), sankapushpi (Convolvulus pluricaulis) etc. if any clinical condition is the contributory factor for insomnia, and it has to be managed in an effective manner. The medhya drugs which have the effect on Vatha and Pitta dosha are quite effective in insomnia. The approach is to relieve the individual from all sorts of stressor and to achieve the state of tranquility, leading to deep relaxation, which ultimately leads to the normalization and stabilization of the mind.

Behavioral therapies such as stimulus control, relaxation, sleep restriction therapy, cognitive and behavioral therapies are beneficial as per reported trials. Cognitive behavioral therapy also produces much better result while managing primary insomnia.

Certain drugs such as Somalata can helps to promote sleep, peculiarly in psychiatric illness as per studies. Steam volatile fraction of the roots and rhizomes of Acorus calamus prolongs the sleeping time, Withania somnifera and Bacopa monnieri posess anti stress properties and Methanol Extract of Hemidesmus indicus possess most of the pharmacological activities characteristic of minor tranquilizers, as per reported experimental studies.

\section{Conclusion}

Insomnia is a very crucial condition and is so much prevalent in the society. It is affecting the quality of life of the affected and is ontributory to much other illness. The modern pharmacological agents are having their own limitations as per reported studies. Ayurveda is a medical branch giving utmost preference in correcting the physiological aspects such as sleep. The vivid Ayurvedic vocabulary is capable of addressing issues such as insomnia to a great extend and can really contribute to the whole medical world. Evidence based studies in this area is the need of the hour and has to be enhanced for the benefit of the affected. 